Article

\title{
Diagnosis Method for Li-Ion Battery Fault Based on an Adaptive Unscented Kalman Filter
}

\author{
Changwen Zheng ${ }^{(\mathbb{D})}$, Yunlong Ge, Ziqiang Chen * (1), Deyang Huang, Jian Liu and Shiyao Zhou \\ State Key Laboratory of Ocean Engineering, Collaborative Innovation Center for Advanced Ship \\ and Deep-sea Exploration, Shanghai Jiao Tong University, Shanghai 200240, China; zcw567@sjtu.edu.cn (C.Z.); \\ gyl19930522@sjtu.edu.cn (Y.G.); 1021226087@sjtu.edu.cn (D.H.); 1262260659@sjtu.edu.cn (J.L.); \\ 569364453@sjtu.edu.cn (S.Z.) \\ * Correspondence: chenziqiang@sjtu.edu.cn; Tel.: +86-21-3420-5001
}

Received: 24 October 2017; Accepted: 4 November 2017; Published: 9 November 2017

\begin{abstract}
The reliability of battery fault diagnosis depends on an accurate estimation of the state of charge and battery characterizing parameters. This paper presents a fault diagnosis method based on an adaptive unscented Kalman filter to diagnose the parameter bias faults for a Li-ion battery in real time. The first-order equivalent circuit model and relationship between the open circuit voltage and state of charge are established to describe the characteristics of the Li-ion battery. The parameters in the equivalent circuit model are treated as system state variables to set up a joint state and parameter space equation. The algorithm for fault diagnosis is designed according to the estimated parameters. Two types of fault of the Li-ion battery, including internal ohmic resistance fault and diffusion resistance faults, are studied as a case to validate the effectiveness of the algorithm. The experimental results show that the proposed approach in this paper has effective tracking ability, better estimation accuracy, and reliable diagnosis for Li-ion batteries.
\end{abstract}

Keywords: battery fault diagnosis; battery management system; parameters estimation; state of charge estimation; adaptive unscented Kalman filter

\section{Introduction}

With the various applications of Li-ion batteries, an effective battery management system (BMS) has become an essential part of the battery system in electrical vehicles and land-based distributed energy storage systems [1]. As an important part of a BMS, battery fault diagnosis techniques have attracted much more attention in recent years [2-5]. For battery cells, as the basic unit of battery packs, the faults of the cell are difficult to detect directly. However, the faults of the cell would cause the variation of internal parameters: leakage of electrolyte will change the polarization characteristics, poor internal connections will lead to the increase of ohmic resistance, etc. Therefore, the real-time estimation of cells is of great importance for system identification, fault detection and isolation, and fault-tolerant control for BMSs.

At present, battery parameter estimation is treated as an effective approach for state of charge (SoC) estimation of batteries [6-15]. Zhang et al. [6] and He et al. [7] implemented an Extended Kalman Filter (EKF) algorithm based on battery parameters estimation to support the estimation of SoC, and achieved a good result in estimation accuracy. The Gauss-Newton method was introduced to iteratively solve battery internal parameters on SoC estimation, and the proposed method has a relatively low computational complexity [8]. Liu et al. [9] suggested a dual Particle Filter estimator based on a temperature-compensated model to estimate battery parameters, and considered the robustness of estimation against temperature changes and noises. Sun et al. [10,11] proposed a recursive least square method (RLSM) with a constant fading factor to identify time-varying battery parameters 
which could accurately capture the dynamic behavior of voltage. Parameter variations could then be linked with battery faults. Chen et al. [16] developed an accurate probabilistic-based framework by using large deviation principles to analyze the reliability of battery fault diagnosis, and a series of simulations and experiments were conducted to validate the effectiveness of the proposed framework. Zhang et al. [17] and Zheng et al. [18] analyzed the capacity degradation of battery packs in a series connection by a recursive-least-squares algorithm with restricted memory and constraints. The battery faults could be distinguished through simulation and experimental validation. Dey et al. [19] modeled electrochemical faults as parametric faults, and multiple sliding observers were incorporated in the diagnostic scheme. The effectiveness of detection, isolation, and estimation of parametric faults were tested via simulation studies. Some works applied parameter estimation-based diagnosis approaches into battery-related applications such as hybrid electric vehicles or Hybrid Energy Storage Systems (HESS) [20,21], which strongly proved the applicability of parameter estimation in Li-ion battery systems. However, all the works mentioned above are limited with solving constant or slow-varying parameters. The parameters of faulty batteries may change quickly or vary in a wide range in some cases. Therefore, these approaches may result in misdiagnosis in such cases.

For improving the reliability of diagnosis, Zhou et al. [22] proposed an extended Kalman filter (EKF) based strong tracking filter (STF) with a time-varying fading factor. STF has strong robustness in dealing with the uncertainty of system models and has a strong tracking ability for the sudden change of parameters or state. Therefore, STF is compatible with real-time estimation on time-varying parameters or state in a wide range. Wang et al. [23] combined STF with an unscented Kalman Filter (UKF) into an adaptive unscented Kalman filter (AUKF) algorithm. The AUKF not only inherits strong robustness from STF, but also overcomes the limits of STF in solving nonlinear problems and simplifies the complexity without calculating a Jacobian matrix. Thus, AUKF is suitable for high-order nonlinear problems and has been successfully applied in engine state estimation and Global Positioning System (GPS) navigation $[24,25]$.

In this paper, the AUKF algorithm is applied to accurately diagnose the parameter bias faults of a Li-ion battery for the first time. Due to the strong nonlinearity and uncertainty, parameters were considered as states of the system. Through the AUKF algorithm, the parameters of the Li-ion battery could be tracked in real time, and the parameters and state could be jointly estimated. Therefore, the faults of the Li-ion battery could be identified and isolated based on the AUKF algorithm according to the different parameter estimation values.

This paper is organized as follows. Section 2 is the battery modeling. In Section 3, the joint parameter estimation method is described and the robustness is analyzed. In Section 4, the parametric diagnosis approach is proposed. In Section 5, experiments are conducted to validate the effectiveness and reliability of the proposed diagnosis algorithm in solving parametric faults of li-ion batteries. Some concluding remarks are provided in Section 6.

\section{Battery Modeling}

Among the present battery modeling approaches, the equivalent circuit model (ECM), electrochemical model, and artificial neural network (ANN) are most commonly used models to capture battery electrical characteristics [26]. In this paper, to compromise computational complexity and modeling accuracy, a simple one-order resistance-capacitance $(R C)$ network ECM is used to describe the battery characteristics. The model is constructed using several components, including an open circuit voltage source $V_{o c v}$, an ohmic resistance $R_{0}$, and one $R C$ network $R_{p}-C_{p}$. The schematic of the ECM is plotted in Figure 1. The ECM differential equations can be expressed as

$$
\begin{gathered}
\dot{v}_{p}(t)=\frac{-1}{R_{p} C_{p}} v_{p}(t)+\frac{1}{C_{p}} i(t) \\
v(t)=v_{o c v}+v_{p}(t)+i(t) R_{0}
\end{gathered}
$$


where $R_{p}$ is the polarize internal resistance; $C_{p}$ is the polarize capacitance; $i(t)$ is the current; $v(t)$ is the terminal voltage of the battery; and $v_{o c v}$ is the open circuit voltage $(\mathrm{OCV})$, which is a nonlinear function of the SoC as described by $v_{o c v}=g(\mathrm{SoC})$. The nonlinear function is utilized to fit the OCV-SoC curve extracted from experimental data. The SoC is calculated as follows:

$$
s(t)=s\left(t_{0}\right)+\int_{t_{0}}^{t} \frac{1}{Q_{0}} i(\tau) d \tau
$$

where $Q_{0}$ is the maximum available capacity. The nonlinear relationship between $\mathrm{OCV}$ and SoC is variable with the temperature. Three OCV-SoC curves in $5{ }^{\circ} \mathrm{C}, 20^{\circ} \mathrm{C}$, and $50{ }^{\circ} \mathrm{C}$ have been obtained from the experimental data, and are shown in Figure 2. Since $V_{o c v}$ is insensitive to temperature, the nonlinear function $g(S o C)$ is fitted with a 7-order polynomial function:

$$
v_{o c v}=397.4 s^{7} v_{o c v}-1434 s^{6}+2093 s^{5}-1583 s^{4}+661.2 s^{3}-150.5 s^{2}+17.38 s+2.417
$$

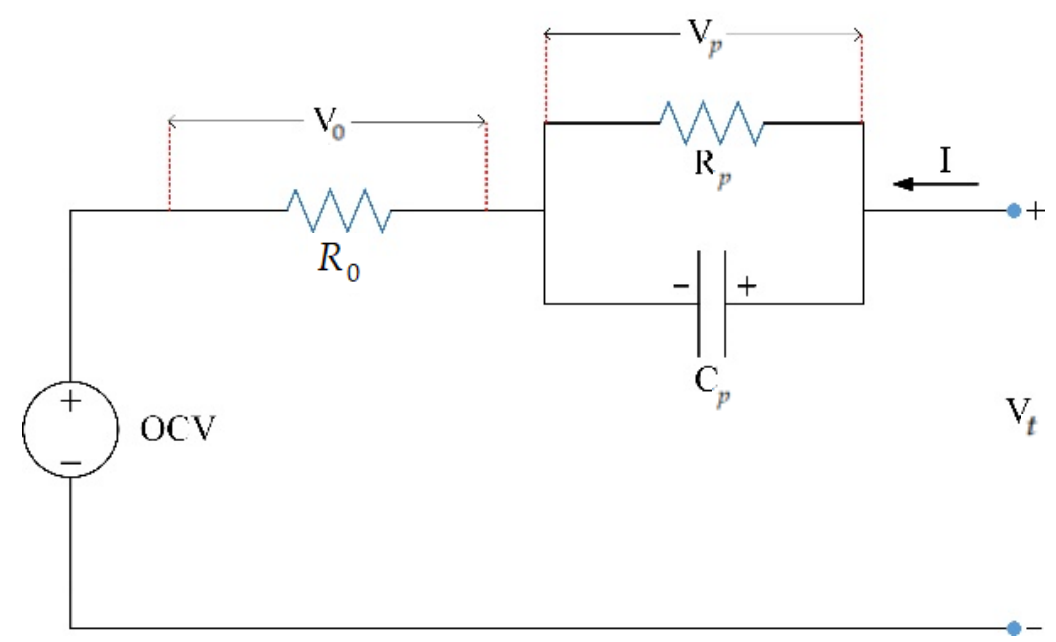

Figure 1. First-order equivalent circuit model of Li-ion battery.

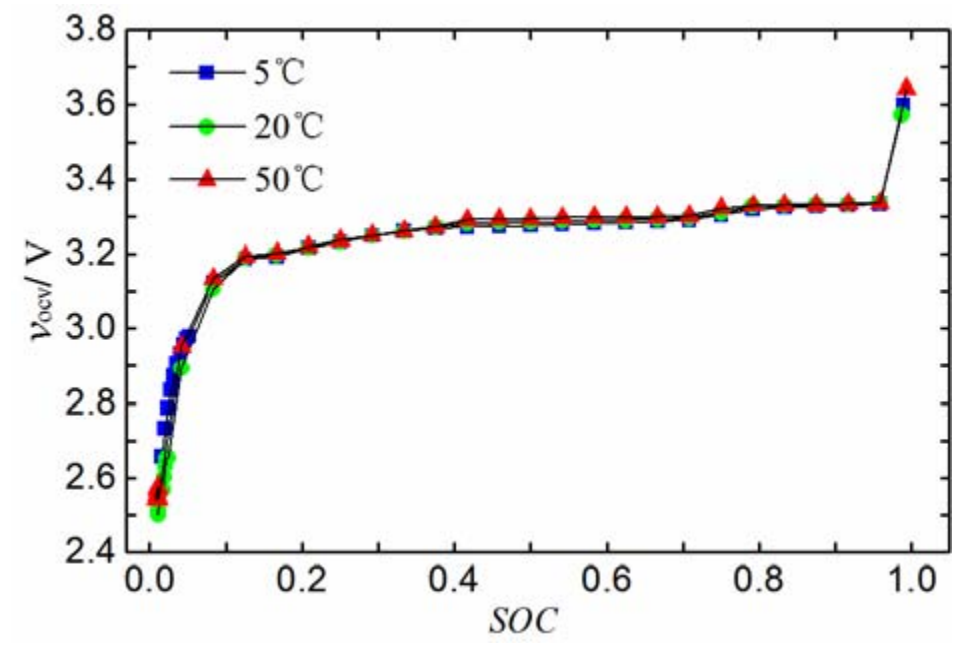

Figure 2. OCV-SoC characteristic mapping curve in different temperature. OCV: open circuit voltage; SoC: state of charge. 


\section{Parameters Estimation}

\subsection{Joint State Space Description}

The main characterizing parameters that affect Li-ion battery performance are $C_{p}, R_{p}, R_{0}$. Therefore, an extended state vector is defined as $\mathrm{x}_{k}=\left[s_{k}, v_{p(k)}, C_{p}, R_{p}, R_{0}\right]$. The steady part of the characterizing parameters is used as a state equation due to the unknown change rule of parameters. The discrete equation can be obtained from (1)-(4):

$$
\begin{aligned}
& \left\{\begin{array}{l}
\mathbf{x}_{k+1}=\mathbf{A}\left(\mathbf{x}_{k}\right) \mathbf{x}_{k}+\mathbf{B}\left(\mathbf{x}_{k}\right) i_{k}+\mathbf{r}_{k} \\
\mathbf{v}_{k+1}=h\left(\mathbf{x}_{k+1}, i_{k+1}\right)+\mathbf{e}_{k+1}
\end{array}\right. \\
& \left\{\begin{array}{l}
\mathrm{A}\left(\mathrm{x}_{k}\right)=\operatorname{diag}\left[1, \exp \left(-T / R_{p} C_{p}\right), 1,1,1\right] \\
\mathrm{B}\left(\mathrm{x}_{k}\right)=\left[T / Q_{0}, R_{p}\left[1, \exp \left(-T / R_{p} C_{p}\right)\right], 0,0,0\right]^{T}
\end{array}\right. \\
& h\left(\mathbf{x}_{k+1}, i_{k+1}\right)=g\left(s_{k+1}\right)+v_{(p) k+1}+i_{k+1} R_{0} \\
& E\left\{r_{k}[l] r_{k}^{T}[l]\right\}=\left\{\begin{array}{l}
Q, l=m \\
0, l \neq m
\end{array}\right. \\
& E\left\{e_{k}[l] e_{k}^{T}[l]\right\}=\left\{\begin{array}{l}
R, l=m \\
0, l \neq m
\end{array}\right.
\end{aligned}
$$

where $\mathbf{v}_{k}$ is the output of the system, $i_{k}$ is the current, $r_{k}$ is the process noise, $e_{k}$ is the measurement noise, and $T$ is the sampling time. Both $r_{k}$ and $e_{k}$ are assumed to be independent, with zero mean value Gaussian white noise with covariance matrix $Q$ and $R$ as (8) and (9), respectively.

\subsection{Joint Estimation Approach}

Traditional UKF has shown good adaptability to nonlinear problems, but has poor robustness. Thus, UKF has poor ability to track abrupt states. As the system becomes stable, the Kalman gain matrix $\mathbf{K}_{k+1}$ approaches 0 . If the system suddenly changes at this moment, the $\mathbf{K}_{k+1} \varepsilon_{k+1}$ is still rather small although the residual $\varepsilon_{k+1}$ continues to increase, and the estimated value of the next time step is unchanged.

STF uses a time-varying fading factor $\lambda_{k+1}$ to adaptively adjust the state prediction covariance as follows [22]:

$$
\mathbf{P}_{k+1 \mid k}=\lambda_{k+1} \mathbf{A}\left(\mathbf{x}_{k}\right) \mathbf{P}_{k \mid k} \mathbf{A}\left(\mathbf{x}_{k}\right)^{T}+\mathbf{Q}
$$

where

$$
\begin{gathered}
\lambda_{k+1}=\left\{\begin{array}{ll}
\lambda_{0}, & \lambda_{0} \geq 1 \\
1, & \lambda_{0}<1^{\prime}
\end{array} \quad \lambda_{0}=\frac{\operatorname{tr}\left(\mathbf{N}_{k+1}\right)}{\operatorname{tr}\left(\mathbf{M}_{k+1}\right)}\right. \\
\mathbf{N}_{k+1}=\mathbf{V}_{k+1}-\mathbf{H}_{k+1} \mathbf{Q} \mathbf{H}_{k+1}{ }^{T}-\eta \mathbf{R} \\
\mathbf{M}_{k+1}=\mathbf{H}_{k+1} \mathbf{P}_{k+1 \mid k} \mathbf{H}_{k+1}^{T}+\mathbf{R}-\mathbf{V}_{k+1}+\mathbf{N}_{k+1} \\
\mathbf{V}_{k+1}=\left\{\begin{array}{cl}
\varepsilon_{1} \varepsilon_{1}^{T}, & k=0 \\
\frac{\rho \mathbf{V}_{k}+\varepsilon_{k+1} \varepsilon_{k+1}}{1+\rho}, & k \geq 1
\end{array}\right.
\end{gathered}
$$


While implementing $\lambda_{k+1}$ into UKF, $\mathbf{H}_{k+1}$ should be expressed as an equivalent form of output prediction covariance $\mathbf{P}_{\hat{\mathbf{v}}_{k+1}}$ and cross-covariance $\mathbf{P}_{\hat{\mathbf{x}}_{k+1} \hat{\mathbf{v}}_{k+1}}$, due to

$$
\begin{aligned}
\mathbf{P}_{\hat{\mathbf{v}}_{k+1}}=E\left[\left(\mathbf{v}_{k+1}-\hat{\mathbf{v}}_{k+1 \mid k}\right)\left(\mathbf{v}_{k+1}-\hat{\mathbf{v}}_{k+1 \mid k}\right)^{T}\right] \\
=E\left\{\left[\mathbf{H}_{k+1}\left(\mathbf{x}_{k+1}-\hat{\mathbf{x}}_{k+1 \mid k}\right)+\mathbf{e}_{k+1}\right]\left[\mathbf{H}_{k+1}\left(\mathbf{x}_{k+1}-\hat{\mathbf{x}}_{k+1 \mid k}\right)+\mathbf{e}_{k+1}\right]^{T}\right\} \\
=\mathbf{H}_{k+1} E\left[\left(\mathbf{x}_{k+1}-\hat{\mathbf{x}}_{k+1 \mid k}\right)\left(\mathbf{x}_{k+1}-\hat{\mathbf{x}}_{k+1 \mid k}\right)^{T}\right] \mathbf{H}_{k+1}^{T}+E\left[\mathbf{e}_{k+1} \mathbf{e}_{k+1}^{T}\right] \\
=\mathbf{H}_{k+1} \mathbf{P}_{k+1 \mid k} \mathbf{H}_{k+1}{ }^{T}+\mathbf{R} \\
\mathbf{P}_{\hat{\mathbf{x}}_{k+1} \hat{\mathbf{v}}_{k+1}}=E\left[\left(\mathbf{x}_{k+1}-\hat{\mathbf{x}}_{k+1 \mid k}\right)\left(\mathbf{v}_{k+1}-\hat{\mathbf{v}}_{k+1 \mid k}\right)^{T}\right] \\
=E\left\{\left(\mathbf{x}_{k+1}-\hat{\mathbf{x}}_{k+1 \mid k}\right)\left[\mathbf{H}_{k+1}\left(\mathbf{x}_{k+1}-\hat{\mathbf{x}}_{k+1 \mid k}\right)+\mathbf{e}_{k+1}-\mathbf{R}\right]^{T}\right\} \\
=E\left[\left(\mathbf{x}_{k+1}-\hat{\mathbf{x}}_{k+1 \mid k}\right)\left(\mathbf{x}_{k+1}-\hat{\mathbf{x}}_{k+1 \mid k}\right)^{T}\right] \mathbf{H}_{k+1}^{T} \\
=\mathbf{P}_{k+1 \mid k} \mathbf{H}_{k+1}{ }^{T}
\end{aligned}
$$

Thus, $\mathbf{H}_{k+1}=\mathbf{P}_{\hat{\mathbf{x}}_{k+1} \hat{\mathbf{v}}_{k+1}}{ }^{T} \mathbf{P}_{\hat{\mathbf{v}}_{k+1}}{ }^{-1}$. The equivalent forms of $\mathbf{N}_{k+1}$ and $\mathbf{M}_{k+1}$ in AUKF are shown in (16):

$$
\begin{aligned}
& \mathbf{N}_{k+1}=\mathbf{V}_{k+1}-\eta \mathbf{R}-\mathbf{P}_{\hat{\mathbf{x}}_{k+1} \hat{\mathbf{v}}_{k+1}}{ }^{T} \mathbf{P}_{k+1 \mid k}{ }^{-1} \mathbf{Q} \mathbf{P}_{k+1 \mid k}{ }^{-1} \mathbf{P}_{\hat{\mathbf{x}}_{k+1} \hat{\mathbf{v}}_{k+1}} \\
& \mathbf{M}_{k+1}=\mathbf{P}_{\hat{\mathbf{v}}_{k+1}}-\mathbf{V}_{k+1}+\mathbf{N}_{k+1}
\end{aligned}
$$

AUKF not only has the advantage of UKF, but can also adaptively adjust the values of noise covariance matrices in the estimation process, which makes the residual sequence mutually orthogonal. The impact of historical data is impaired to enhance the tracking ability of the filter. When the parameter bias fault happens, Equation (5) is compatible with the model and the AUKF could be used to estimate the characterizing parameters. The AUKF algorithm has strong robustness against uncertainties and disturbance and its robustness had been verified in [22].

In the AUKF algorithm, a set of $2 n+1$ Sigma points $\left\{\boldsymbol{\xi}_{i}\right\}$ and corresponding weights $W^{m}$ and $W^{c}$ from $n$-dimension state vector $\mathbf{x}$ are generated through unscented transformation:

$$
\begin{aligned}
& \left\{\begin{array}{l}
\xi_{0}=\hat{\mathbf{x}} \\
\xi_{i}=\hat{\mathbf{x}}+(\sqrt{(n+\kappa) \mathbf{P}})_{i^{\prime}} \quad i=1,2, \ldots, n \\
\xi_{i}=\hat{\mathbf{x}}-(\sqrt{(n+\kappa) \mathbf{P}})_{i^{\prime}} \quad i=n+1, n+2, \ldots, 2 n
\end{array}\right. \\
& \left\{\begin{array}{l}
W_{0}^{m}=\kappa /(n+\kappa) \\
W_{0}^{c}=\kappa /(n+\kappa)+\left(1-\alpha^{2}+\beta\right) \\
W_{i}^{m}=W_{i}^{c}=1 /[2(n+\kappa)], \quad i=1,2, \ldots, 2 n
\end{array}\right.
\end{aligned}
$$

where $\kappa=\alpha^{2}(n+k)-n, \alpha$ is a scale factor that can be chosen as $\alpha=1$, and $\beta$ is a factor for improving the accuracy of variance that can be set as $\beta=2$ for Gaussian distribution. The details of the AUKF algorithm are listed in Algorithm 1. 
Algorithm 1. Details of the Adaptive Unscented Kalman Filter (AUKF) Algorithm

\section{Step 1: Initialize}

Initializing estimated state value $\hat{\mathbf{x}}_{0 \mid 0}$ and covariance matrix $\mathbf{P}_{0 \mid 0}$.

Step 2: Predict State and output

Transform Sigma points $\xi_{i, k}$ to $\gamma_{i, k+1 \mid k}$ through (5), one-step estimate state $\hat{\mathbf{x}}_{k+1 \mid k}$, and covariance matrix $\mathbf{P}_{k+1 \mid k}$.

$$
\left\{\begin{array}{l}
\gamma_{i, k+1 \mid k}=\mathbf{A}\left(\boldsymbol{\xi}_{i, k}\right) \boldsymbol{\xi}_{i, k}+\mathbf{B}\left(\boldsymbol{\xi}_{i, k}\right) i_{k} \\
\hat{\mathbf{x}}_{k+1 \mid k}=\sum_{i=0}^{2 n} W_{i}^{m} \gamma_{i, k+1 \mid k} \\
\mathbf{P}_{k+1 \mid k}=\sum_{i=0}^{2 n} W_{i}^{c}\left[\gamma_{i, k+1 \mid k}-\hat{\mathbf{x}}_{k+1 \mid k}\right]\left[\gamma_{i, k+1 \mid k}-\hat{\mathbf{x}}_{k+1 \mid k}\right]^{T}
\end{array}\right.
$$

Predict Sigma points $\boldsymbol{\xi}_{i, k+1 \mid k}$ through (8) and (9) with $\hat{\mathbf{x}}_{k+1 \mid k}$ and $\mathbf{P}_{k+1 \mid k}$. Output prediction can be obtained from (5) with $\boldsymbol{\xi}_{i, k+1 \mid k}$. We calculate the output prediction covariance matrix $\mathbf{P}_{\hat{\mathbf{v}}_{k+1}}$ and cross-covariance $\mathbf{P}_{\hat{\mathbf{x}}_{k+1}} \hat{\mathbf{v}}_{k+1}$ through $\hat{\boldsymbol{v}}_{k+1 \mid k}$ :

$$
\left\{\begin{array}{l}
\hat{\mathbf{v}}_{k+1 \mid k}=\sum_{i=0}^{2 n} W_{i}^{m} h\left(\boldsymbol{\xi}_{i, k+1 \mid k}, i_{k+1}\right) \\
\mathbf{P}_{\hat{\mathbf{v}}_{k+1}}=\sum_{i=0}^{2 n} W_{i}^{c}\left[h\left(\boldsymbol{\xi}_{i, k+1 \mid k}, i_{k+1}\right)-\hat{\mathbf{v}}_{k+1 \mid k}\right]\left[h\left(\boldsymbol{\xi}_{i, k+1 \mid k}, i_{k+1}\right)-\hat{\mathbf{v}}_{k+1 \mid k}\right]^{T} \\
\mathbf{P}_{\hat{\mathbf{x}}_{k+1} \hat{\mathbf{v}}_{k+1}}=\sum_{i=0}^{2 n} W_{i}^{c}\left[\boldsymbol{\xi}_{i, k+1 \mid k}-\hat{\mathbf{x}}_{k+1 \mid k}\right]\left[h\left(\boldsymbol{\xi}_{i, k+1 \mid k}, i_{k+1}\right)-\hat{\mathbf{v}}_{k+1 \mid k}\right]^{T} \\
\boldsymbol{\varepsilon}_{k+1}=\mathbf{v}_{k+1}-\hat{\mathbf{v}}_{k+1 \mid k}
\end{array}\right.
$$

where $\varepsilon_{k+1}$ is the residual. The fading factor $\lambda_{k+1}$ can be calculated by the approaches in [19].

$$
\lambda_{k+1}=\left\{\begin{array}{ll}
\lambda_{0}, & \lambda_{0} \geq 1 \\
1, & \lambda_{0}<1
\end{array}, \quad \lambda_{0}=\frac{\operatorname{tr}\left(\mathbf{N}_{k+1}\right)}{\operatorname{tr}\left(\mathbf{M}_{k+1}\right)}\right.
$$

where

$$
\begin{aligned}
& \left\{\begin{array}{l}
\mathbf{N}_{k+1}=\mathbf{V}_{k+1}-\eta \mathbf{R}-\mathbf{P}_{\hat{\mathbf{x}}_{k+1} \hat{\mathbf{v}}_{k+1}}{ }^{T} \mathbf{P}_{k+1 \mid k}{ }^{-1} \mathbf{Q} \mathbf{P}_{k+1 \mid k}{ }^{-1} \mathbf{P}_{\hat{\mathbf{x}}_{k+1} \hat{\mathbf{v}}_{k+1}} \\
\mathbf{M}_{k+1}=\mathbf{P}_{\hat{\mathbf{v}}_{k+1}}-\mathbf{V}_{k+1}+\mathbf{N}_{k+1}
\end{array}\right. \\
& \mathbf{V}_{k+1}=\left\{\begin{array}{cc}
\varepsilon_{1} \varepsilon_{1}{ }^{T}, & k=0 \\
\frac{\rho \mathbf{V}_{k}+\varepsilon_{k+1} \varepsilon_{k+1}{ }^{T}}{1+\rho}, & k \geq 1
\end{array}\right.
\end{aligned}
$$

where $0<\rho<1$ is the fading factor, and $\rho$ is normally equal to 0.95 .

Modify the prediction of the covariance matrix:

$$
\mathbf{P}_{k+1 \mid k}=\lambda_{k+1} \sum_{i=0}^{2 n} W_{i}^{c}\left[\boldsymbol{\xi}_{i, k+1 \mid k}-\hat{\mathbf{x}}_{k+1 \mid k}\right]\left[\boldsymbol{\xi}_{i, k+1 \mid k}-\hat{\mathbf{x}}_{k+1 \mid k}\right]^{T}
$$

Recalculate Sigma points $\xi_{i, k+1 \mid k}$ with modified $\mathbf{P}_{k+1 \mid k}$ and $\hat{\mathbf{x}}_{k+1 \mid k}$. Modify $\hat{\mathbf{v}}_{k+1 \mid k}, \mathbf{P}_{\hat{\mathbf{v}}_{k+1}}$ and $\mathbf{P}_{\hat{\mathbf{x}}_{k+1} \hat{\mathbf{v}}_{k+1}}$ through (11).

Step 3: Update state

$$
\left\{\begin{array}{l}
\hat{\mathbf{x}}_{k+1 \mid k+1}=\hat{\mathbf{x}}_{k+1 \mid k}+\mathbf{K}_{k+1} \varepsilon_{k+1} \\
\mathbf{K}_{k+1}=\mathbf{P}_{\hat{\mathbf{x}}_{k+1} \hat{\mathbf{v}}_{k+1}} \mathbf{P}_{\hat{\mathbf{v}}_{k+1}}-1 \\
\mathbf{P}_{k+1 \mid k+1}=\mathbf{P}_{k+1 \mid k}-\mathbf{K}_{k+1} \mathbf{P}_{\hat{\mathbf{v}}_{k+1}} \mathbf{K}_{k+1}^{T}
\end{array}\right.
$$

Step 4: Set $k=k+1$, return to Step 2 and start iteration

\section{Diagnosis Approach for Parameter Bias Fault}

Faults can be divided into abrupt-type faults and slow-varying-type faults, according to the variation pattern of parameters [22]. For Li-ion battery cells, the diffusivity and contact ability of the electrolyte will vary when some faults occur, and will result in the variation of the time constant $\tau=R_{p} C_{p}$ and ohmic internal resistance $R_{0}$, respectively. The faults can be detected by calculating the 
standard deviations of two parameters. These standard deviations are compared with some thresholds. A corresponding fault is detected once the standard deviation is beyond a certain threshold. Therefore, we implement the diagnosis on $\tau$ and $R_{0}$, and define

$$
\begin{aligned}
& \boldsymbol{\theta}=\frac{1}{N+1} \sum_{j=0}^{N}\left(\begin{array}{c}
\hat{\tau}_{k-j} \\
\hat{R}_{0(k-j)}
\end{array}\right) \\
& \mathbf{S}_{R_{0}}^{1}=\frac{1}{N} \sum_{j=0}^{N}\left[\hat{R}_{0(k-j)}-\boldsymbol{\theta}_{R_{0}}^{*}\right]^{2} \\
& \mathbf{S}_{\tau}^{1}=\frac{1}{N} \sum_{j=0}^{N}\left[\hat{\tau}_{k-j}-\boldsymbol{\theta}_{\tau}^{*}\right]^{2} \\
& \mathbf{S}_{R_{0}}^{2}=\frac{1}{N} \sum_{j=0}^{N}\left[\hat{R}_{0(k-j)}-\boldsymbol{\theta}_{R_{0}}\right]^{2} \\
& \mathbf{S}_{\tau}^{2}=\frac{1}{N} \sum_{j=0}^{N}\left[\hat{\tau}_{k-j}-\boldsymbol{\theta}_{\tau}\right]^{2}
\end{aligned}
$$

where $N$ is the data window, and $\theta^{*}$ is the normal value of the parameter.

The fault detector is expressed as

$$
\mathbf{S}_{\phi}^{i} \underset{H_{0}}{\stackrel{H_{1}}{\geq}} \varepsilon_{\varphi}^{i}\left(i=1,2 ; \varphi=R_{0}, \tau\right)
$$

where $H_{1}$ means the fault is happening, and $H_{0}$ means no fault. The slow-varying-type fault thresholds and the abrupt-type fault thresholds-which could be chosen by experience or by trial and error tests-are defined as $\varepsilon^{1}$ and $\varepsilon^{2}$, respectively. The fault map is listed in Table 1 according to the fault detector. The diagnosis initializes with the following process, and the diagnosis flow-chart is presented in Figure 3.

Step 1: Set a sequence of parameters within a data window $N$;

Step 2: Extract $\hat{R}_{0(k-j)}$ and $\hat{\tau}_{k-j}$ from the sequence;

Step 3: Calculate $\boldsymbol{\theta}_{R_{0}}^{*}, \mathbf{S}_{R_{0}}^{1}, \mathbf{S}_{\tau}^{1}$ and $\boldsymbol{\theta}_{\tau}^{*}, \mathbf{S}_{R_{0}}^{2}, \mathbf{S}_{\tau}^{2}$, respectively;

Step 4: Start fault detection to detect the faults. If there is a fault, the detector alerts the system and breaks out; if there is no fault, the system moves to $k+1$.

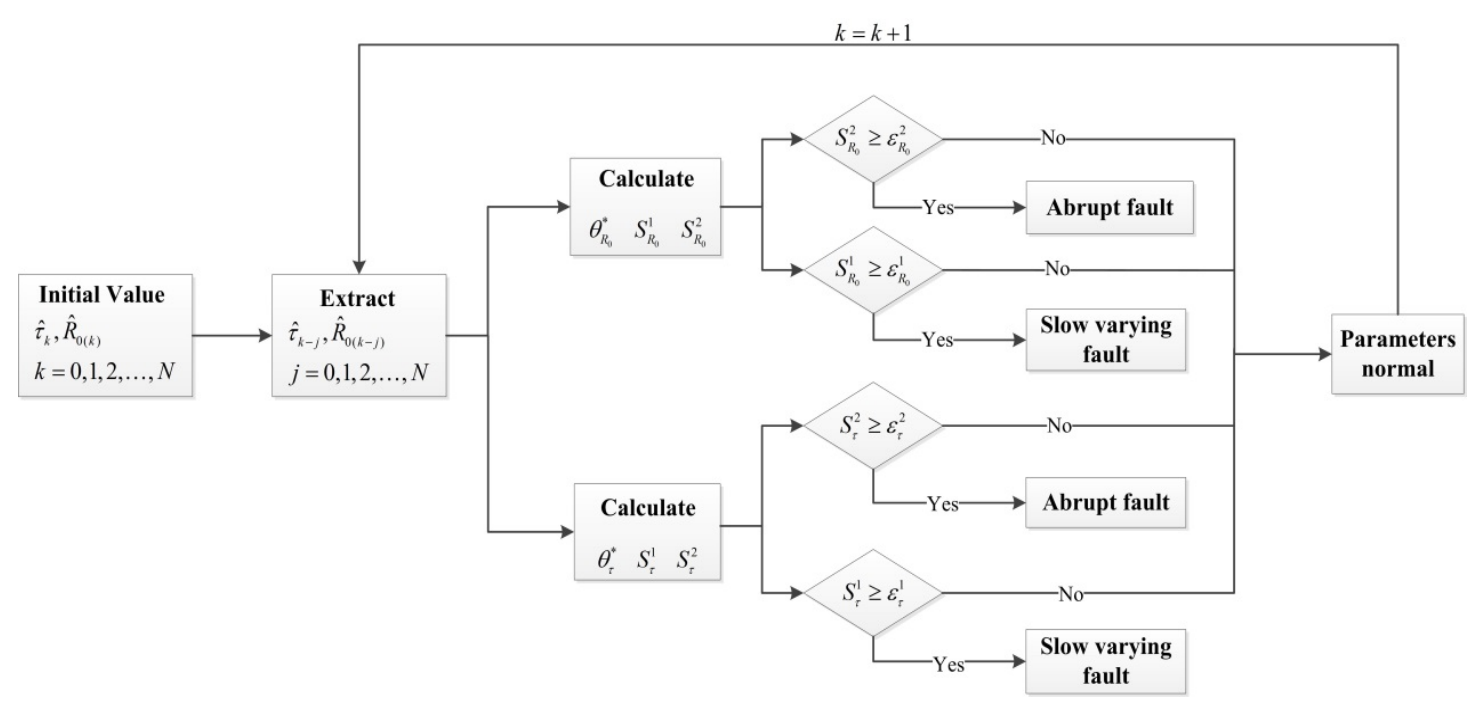

Figure 3. Parameter-biased fault diagnosis process of battery cell. 
Table 1. Fault map.

\begin{tabular}{cc}
\hline$R_{0}$ slow varying fault & $S_{R_{0}}^{1}>\varepsilon_{R_{0}}^{1}$ \\
$R_{0}$ abrupt fault & $S_{\tau}^{1}>\varepsilon_{\tau}^{1}$ \\
$\tau$ slow varying fault & $S_{R_{0}}^{2}>\varepsilon_{R_{0}}^{2}$ \\
$\tau$ abrupt fault & $S_{\tau}^{2}>\varepsilon_{\tau}^{2}$ \\
\hline
\end{tabular}

\section{Experimental Validation}

For verifying the effectiveness of the diagnosis proposed in the paper, the experiment is conducted through simulating the abrupt-type fault and slow-varying-type fault of a Li-ion battery by controlling the battery ambient temperature as a case study. The battery parameters are estimated in real time, and the battery faults are diagnosed by comparing the algorithms of AUKF and UKF in this case. The test bench consists of a BTS- 4000 battery test device, an auxiliary data acquisition channel, a BLH-100 incubator, and an upper PC and monitor software, as shown in Figure 4. The battery type that we have chosen for the experiment is $\mathrm{WX}-14 \mathrm{I} 3320 \mathrm{LiFeO}_{4}$. The specification of the battery is listed in Table 2.

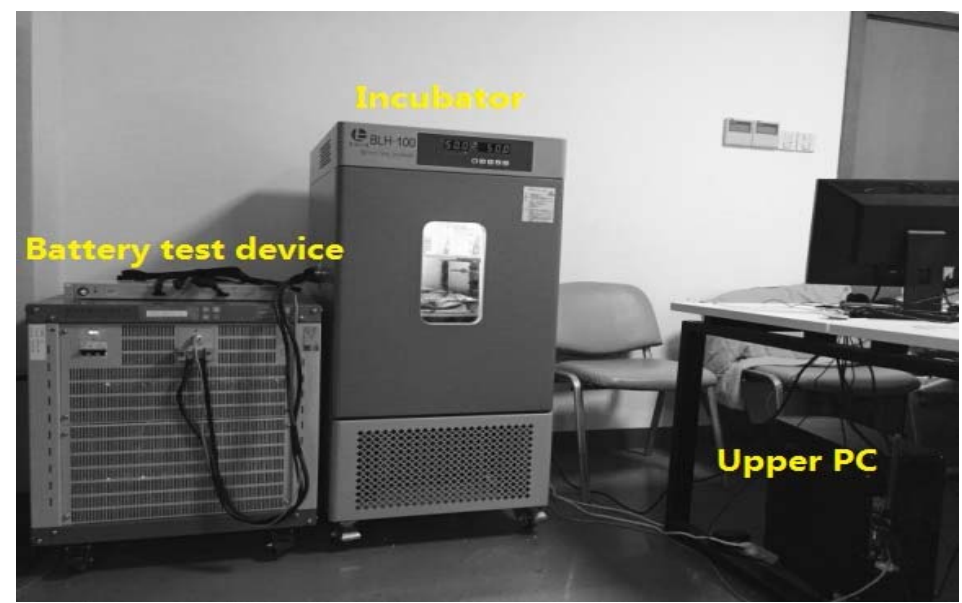

Figure 4. Battery testing bench.

Table 2. Specification of $\mathrm{LiFePO}_{4}$ Power Battery.

\begin{tabular}{cc}
\hline Rated Capacity & $20 \mathrm{Ah}$ \\
Max Charge Voltage & $3.65 \mathrm{~V}$ \\
Min discharge Voltage & $2.5 \mathrm{~V}$ \\
Charge Rate & $1 \mathrm{C}$ \\
Continuous discharge Rate & $3 \mathrm{C}$ \\
\hline
\end{tabular}

The hybrid pulse power characterization (HPPC) test is implemented off-line to identify the $\tau$ and $R_{0}$ at different temperatures and SoCs. Seen from Figure 5 , the results show that $R_{0}$ increases with the decreasing temperature, while $\tau$ varies slightly with the variation of temperature, which is similar to those in [27]. During the fault simulation, the actual value of parameters can be obtained from the real temperature and SoC according to the relationship in Figure 5. Therefore, the validation of AUKF is confirmed. The Federal Urban Driving Schedule (FUDS) is chosen for the battery charge and discharge test and the current profiles are plotted in Figure 6 . The sampling time is $1 \mathrm{~s}$, and the test time is $3600 \mathrm{~s}$. The algorithm initial values are set as follows: initial SoC $s_{0}=0.8$, polarization voltage $v_{p(0)}=-0.03 \mathrm{~V}$. The parameters are chosen from the identification results in $20^{\circ} \mathrm{C}: R_{0}=R_{p}=1 \mathrm{~m} \Omega$, $C_{p}=30 \mathrm{kF}$. 


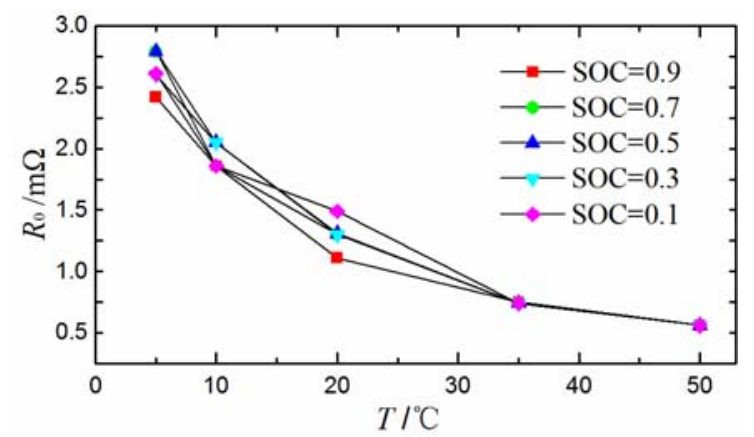

(a)

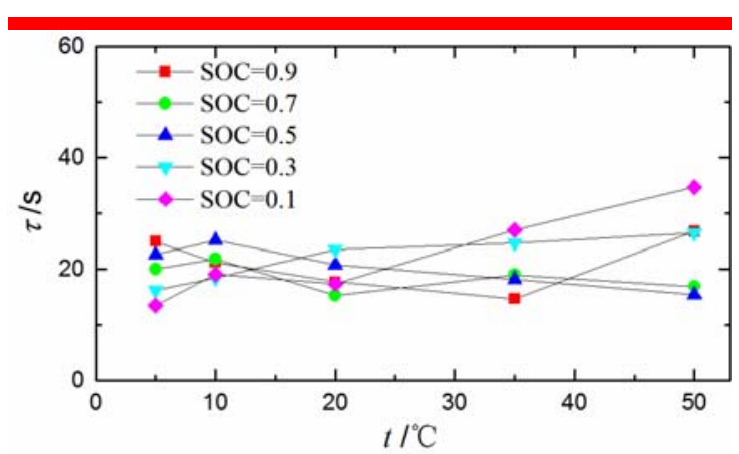

(b)

Figure 5. Off-line identification results of two parameters. (a) Ohmic Resistances $R_{0}$; (b) Time constant $\tau$.

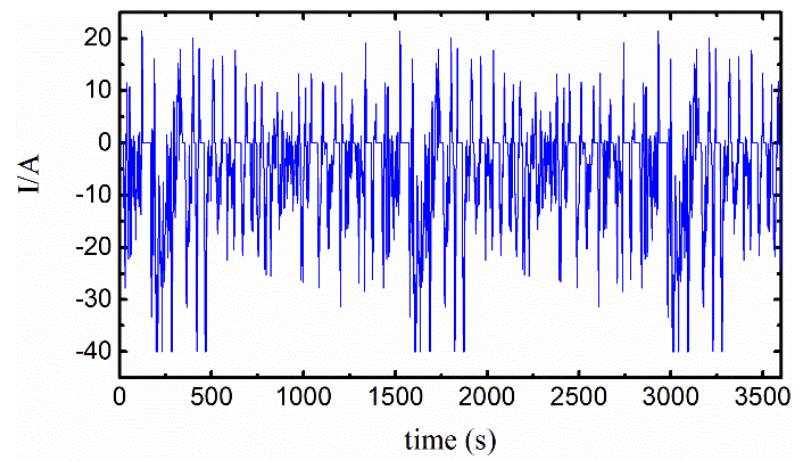

Figure 6. Battery current profile during Federal Urban Driving Schedule (FUDS) cycles.

\subsection{Diagnosis of Slow-Varying-Type Fault}

For simulating the slow-varying-type fault, we dropped the ambient temperature from $50{ }^{\circ} \mathrm{C}$ to $5{ }^{\circ} \mathrm{C}$ in the incubator over a time of $0-3600 \mathrm{~s}$. The measured voltage value is shown in Figure 7 . The results of comparison among the estimated values $R_{0}$ gained using the algorithm of AUKF, the estimated values $R_{0}$ gained using the algorithm of UKF, and the real values, are plotted in Figure 8a. It shows that the trend of $R_{0}$ given by AUKF is the same as the real value. Both algorithms are effective and insensitive with initial values during the estimation before $1000 \mathrm{~s}$. After $3600 \mathrm{~s}$, the AUKF estimation value reaches $2.88 \mathrm{~m} \Omega$ with a relative error of $15.3 \%$, while the UKF estimation value reaches $1.62 \mathrm{~m} \Omega$ with a relative error of $43.8 \%$ which shows poor tracking ability. It indicates that the algorithm of AUKF has better robustness than UKF.

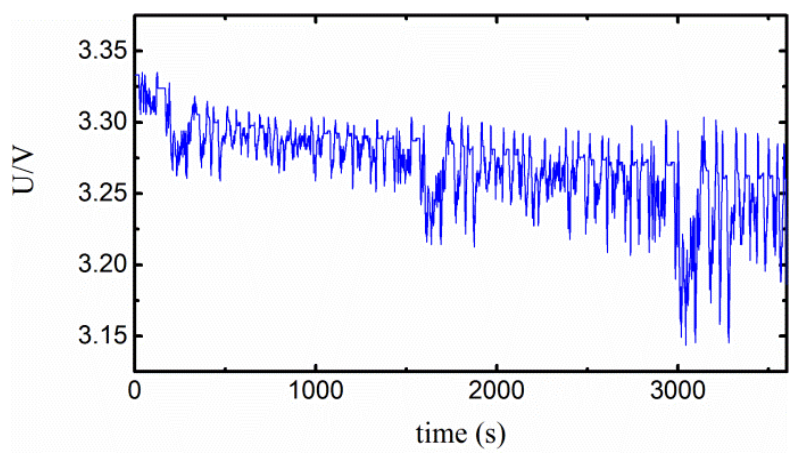

Figure 7. Terminal voltage of battery with parameter slow-varying fault. 
The AUKF and UKF have different estimation trends of time constant $\tau$ after $800 \mathrm{~s}$, as shown in Figure $8 \mathrm{~b}$. The relative estimation error of $\tau$ from using the AUKF after $3600 \mathrm{~s}$ is $30.8 \%$ compared with the real value of $\tau$. The high error is due to the relatively low ECM accuracy, poor identifiability of polarization resistance and polarization capacitance, etc. However, the UKF estimation is divergent after $800 \mathrm{~s}$.

To further discuss the tracking ability of AUKF, the comparison of terminal voltage estimation error $\varepsilon$ of the battery between the two algorithms is plotted in Figure $8 \mathrm{c}$. The adaptive fading factor in AUKF can adjust the covariance matrices and makes the residual sequences orthogonal. Thus, the absolute value of $\varepsilon$ could be restricted within $15 \mathrm{mV}$, while the residual $\varepsilon$ estimated by UKF is divergent and is fluctuating obviously after $2000 \mathrm{~s}$. The maximum residual reaches $40 \mathrm{mV}$, which indicates that the UKF is less effective than the AUKF.

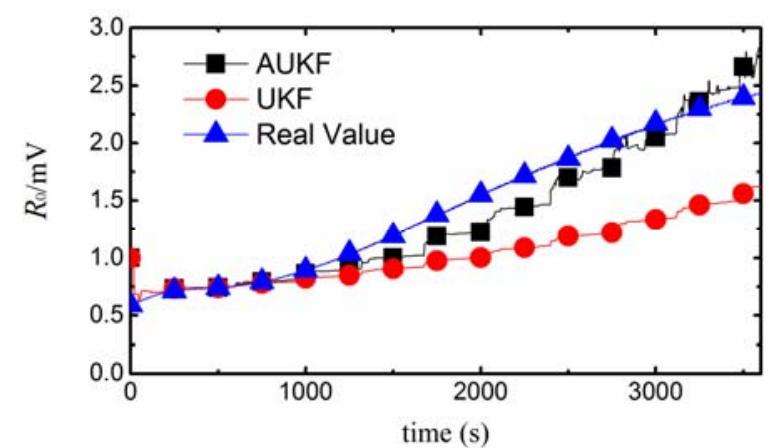

(a)

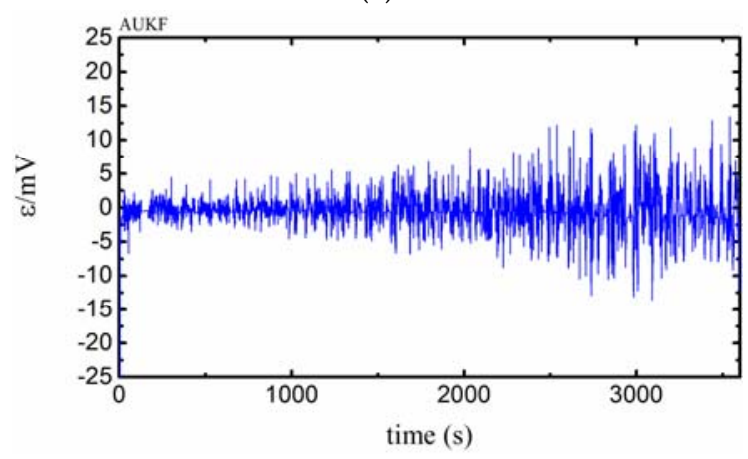

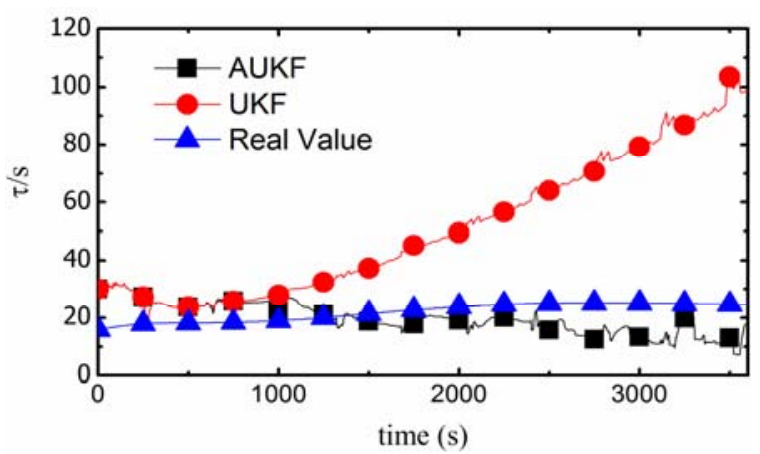

(b)

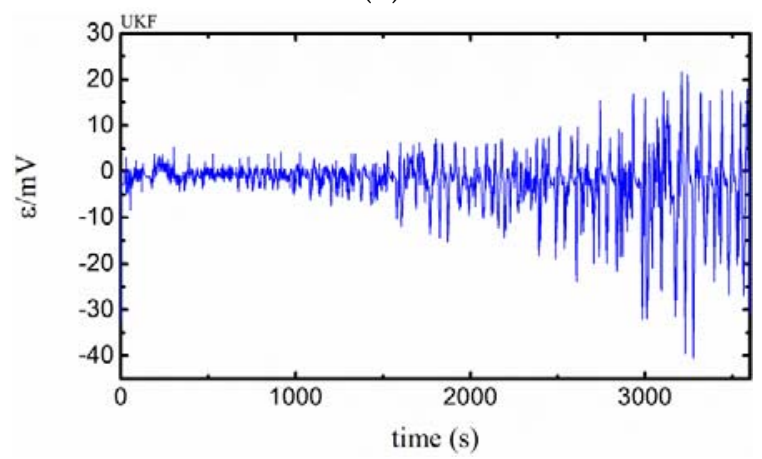

(c)

Figure 8. Estimation results of parameter slow-varying type fault. (a) Comparison of Ohmic resistance estimation; (b) Comparison of time constant estimation; (c) Comparison of voltage residual.

For evaluating the parameter diagnosis, an example is taken as follows. By setting the data window $N=50$, normal parameter $\theta^{*}=(20,0.5)^{T}$, and thresholds $\varepsilon^{1}=\left(20^{2}, 1^{2}\right)^{T}$, the diagnosis results for the slow-varying-type fault are listed in Table 3. It shows that the AUKF-based diagnosis method diagnoses the contact fault at $2311 \mathrm{~s}$, which is faster than the UKF-based algorithm of $3585 \mathrm{~s}$ by $78.4 \%$. Moreover, the UKF-based algorithm misdiagnoses the contact fault at $1748 \mathrm{~s}$ while the AUKF one gives a normal result. Therefore, the AUKF-based diagnosis has better accuracy and is more reliable than that of UKF. 
Table 3. Diagnosis Result of Slow-Varying-Type Fault.

\begin{tabular}{ccccc}
\hline \multirow{2}{*}{ Item } & \multicolumn{2}{c}{ Contact Fault } & \multicolumn{2}{c}{ Diffusion Fault } \\
\cline { 2 - 5 } & AUKF & UKF & AUKF & UKF \\
\hline Detection time/s & 2311 & 3585 & - & 1748 \\
Diagnosis result & Faulty & Faulty & Normal & Normal \\
Real fault situation & Fault $(1960 \mathrm{~s})$ & \multicolumn{2}{c}{ Normal } \\
\hline
\end{tabular}

\subsection{Diagnosis of Abrupt Type Fault}

To simulate the parameter of an abrupt-type fault, the battery cell was maintained in a $50{ }^{\circ} \mathrm{C}$ constant temperature for $1500 \mathrm{~s}$ through the incubator. Then, we cooled it down to $5^{\circ} \mathrm{C}$ and maintained the temperature until $3600 \mathrm{~s}$. The terminal voltage profile is shown in Figure 9. Seen from Figure 10a, the estimated value $R_{0}$ by using each of the two algorithms-AUKF and UKF-converges to $0.7 \mathrm{~m} \Omega$ before $1300 \mathrm{~s}$. Compared with the real value of $0.61 \mathrm{~m} \Omega$, the relative error is $14.3 \%$. After $1800 \mathrm{~s}$, the UKF shows poor tracking ability on the abrupt-type fault. The estimated value of AUKF and UKF reaches to $2.57 \mathrm{~m} \Omega$ and $1.74 \mathrm{~m} \Omega$, with relative error of $8.4 \%$ and $26.6 \%$, respectively. The estimated value $\tau$ by using each of the two algorithms converges to $19 \mathrm{~s}$ with a relative error of $25 \%$ before $1300 \mathrm{~s}$. Being the same as the diagnosis results of the slow-varying-type fault, the AUKF converges around $18 \mathrm{~s}$ while the UKF is still divergent, and the final relative errors of AUKF and UKF are $16.9 \%$ and $372 \%$, respectively, as shown in Figure 10b.

For the abrupt-type fault, the distinct residual comparison between the AUKF and the UKF is presented in Figure 10c. It indicates residual $\varepsilon$ increases abruptly after $1300 \mathrm{~s}$. The AUKF can rapidly adjust $\varepsilon$ within $15 \mathrm{mV}$, while the UKF will continue to enlarge the residual after $1300 \mathrm{~s}$, and the residual reaches near $30 \mathrm{mV}$ after $2500 \mathrm{~s}$. The trend of divergence shows that the UKF is invalid in such a case.

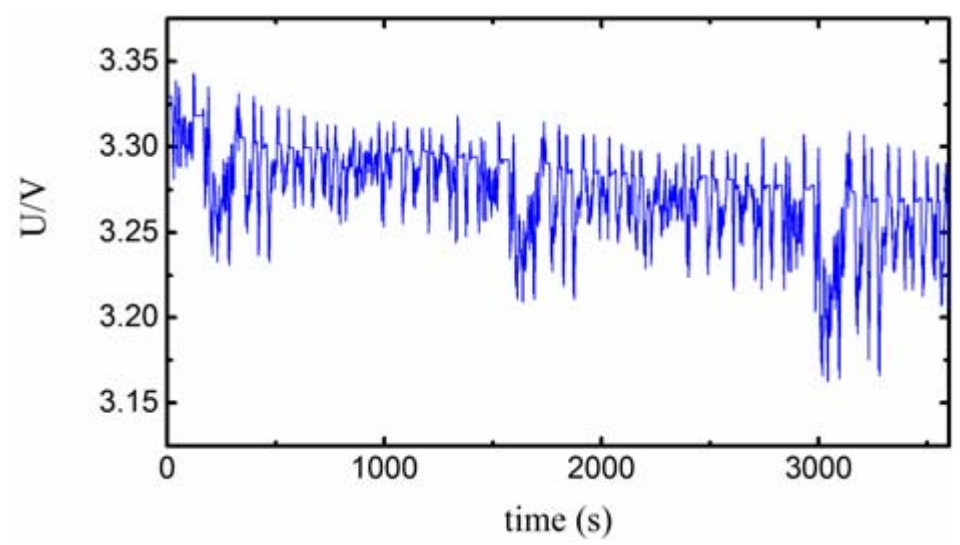

Figure 9. Terminal voltage of battery with abrupt-type fault. 


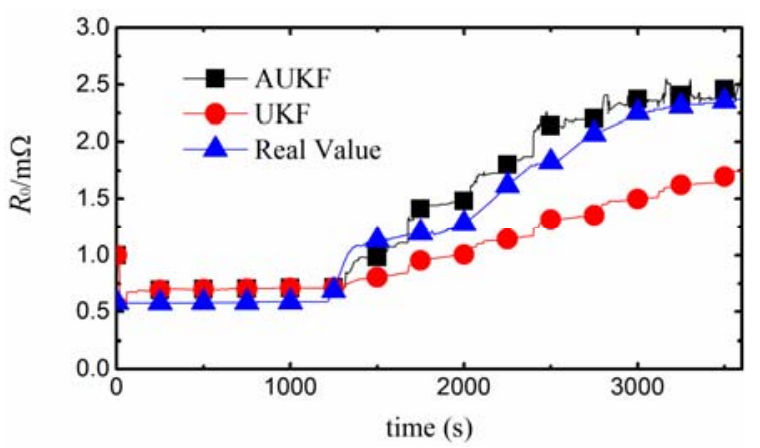

(a)

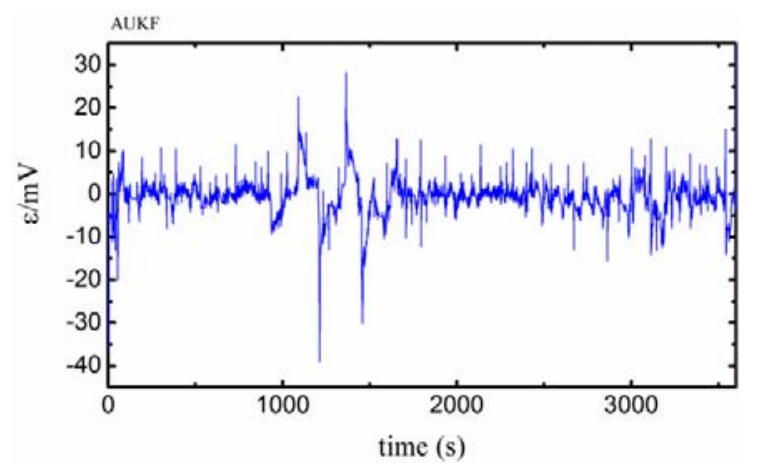

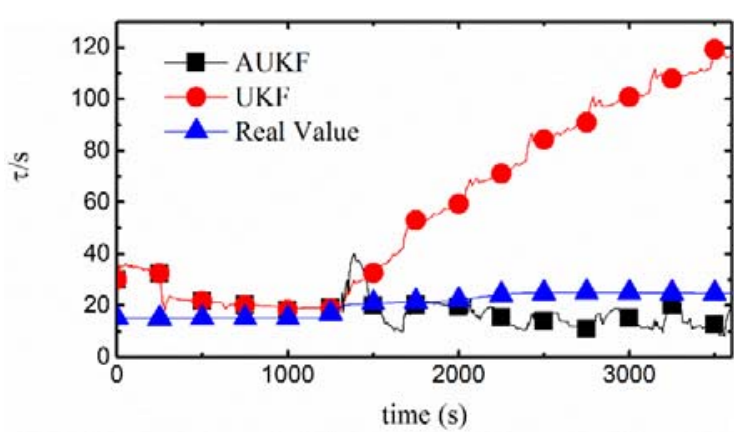

(b)

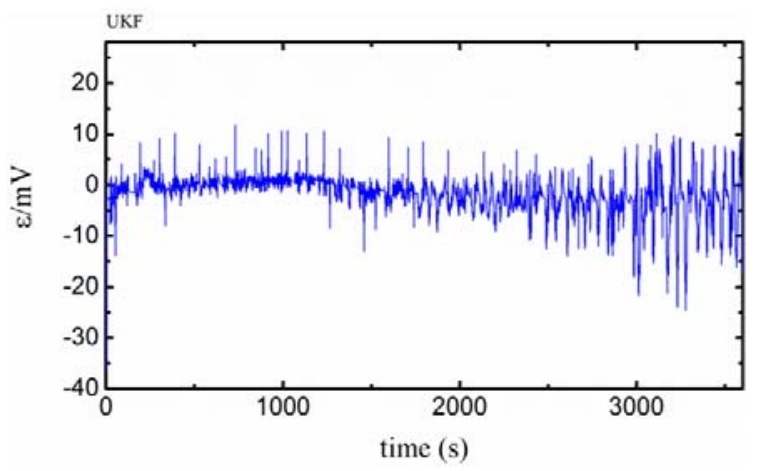

(c)

Figure 10. Estimation results of parameter abrupt-type fault. (a) Comparison of estimated ohmic resistance; (b) Comparison of estimated time constant; (c) Comparison of voltage residual.

The diagnosis settings are the data window $N=100$ and the threshold $\varepsilon^{2}=\left(5^{2}, 0.1^{2}\right)^{T}$. The diagnosis results are listed in Table 4 . The contact fault is detected at 1715 s by the AUKF, while the UKF misdiagnoses the fault as normal. For the diffusion type of fault, the AUKF misdiagnoses the normal as fault at $1380 \mathrm{~s}$, while the UKF diagnoses it as normal. The misdiagnosis by AUKF is due to the fact that the single fading factor is unable to adjust each state variable independently. Because of the effect of the estimated value $R_{0}$, a large fluctuation in the estimated value $\tau$ happens at $1400 \mathrm{~s}$. This problem could be solved by introducing multiple fading factors [22].

Table 4. Diagnosis result of Parameter Abrupt-Type Fault.

\begin{tabular}{ccccc}
\hline \multirow{2}{*}{ Item } & \multicolumn{2}{c}{ Contact Fault } & \multicolumn{2}{c}{ Diffusion Fault } \\
\cline { 2 - 5 } & AUKF & UKF & AUKF & UKF \\
\hline Detection time/s & 1715 & - & 1380 & - \\
Diagnosis result & Faulty & Normal & Faulty & Normal \\
Real fault situation & \multicolumn{2}{c}{ Fault $(1300 \mathrm{~s})$} & \multicolumn{2}{c}{ Normal } \\
\hline
\end{tabular}

In general, the diagnosis method based on AUKF for Li-ion battery faults offers great advantages in accuracy, reliability, and computational effort over the one based on UKF.

\section{Conclusions}

This paper proposes an adaptive unscented Kalman filter based parameter estimation algorithm and fault diagnosis method for Li-ion batteries. The parameters estimated by the adaptive unscented Kalman filter are utilized to diagnose two types of parameter fault-slow-varying-type faults and abrupt-type faults-through defining some fault threshold values. The experimental results show that 
the algorithm of the adaptive unscented Kalman filter has better tracking ability and higher accuracy on battery fault parameters than the unscented Kalman filter. Therefore, the adaptive unscented Kalman filter based diagnosis method proposed in this paper has better effectiveness in diagnosis, higher reliability, and more moderate computational effort. The methodologies of this paper can be extended to different battery types and battery packs for diagnosing the faults of battery systems. It is worth exploring such an extension for providing a relatively new train of thought for real-time fault diagnosis of complex battery systems.

Acknowledgments: The authors gratefully acknowledge the support provided by National Natural Science Foundation of China (No. 51677119).

Author Contributions: Ziqiang Chen provided guidance and supervision. Changwen Zheng implemented the main research, checked and discussed the results, and wrote the paper. Yunlong Ge, Deyang Huang, Jian Liu, and Shiyao Zhou performed the simulation and experiment, and checked the paper. All authors read and approved the final manuscript.

Conflicts of Interest: The authors declare no conflict of interest.

\section{References}

1. Andrea, D. Battery Management Systems for Large Lithium-Ion Battery Packs; Artech House: Norwood, MA, USA, 2010.

2. Sidhu, A.; Izadian, A.; Anwar, S. Adaptive nonlinear model-based fault diagnosis of Li-ion batteries. IEEE Trans. Ind. Electron. 2015, 62, 1002-1011. [CrossRef]

3. Xia, B.; Shang, Y.; Nguyen, T.; Mi, C. A correlation based fault detection method for short circuits in battery packs. J. Power Sources 2017, 337, 1-10. [CrossRef]

4. Chen, W.; Chen, W.T.; Saif, M.; Li, M.F.; Wu, H. Simultaneous fault isolation and estimation of lithium-ion batteries via synthesized design of Luenberger and learning observers. IEEE Trans. Control Syst. Technol. 2014, 22, 290-298. [CrossRef]

5. Chen, Z.; Lin, F.; Wang, C.S.; Wang, L.Y.; Xu, M. Active diagnosability of discrete event systems and its application to battery fault diagnosis. Int. J. IEEE Trans. Control Syst. Technol. 2014, 22, 1892-1898. [CrossRef]

6. Zhang, X.; Wang, Y.; Yang, D.; Chen, Z. An on-line estimation of battery pack parameters and state-of-charge using dual filters based on pack model. Energy 2016, 115, 219-229. [CrossRef]

7. He, H.; Xiong, R.; Zhang, X.; Sun, F.; Fan, J.X. State-of-charge estimation of the lithium-ion battery using an adaptive extended Kalman filter based on an improved Thevenin model. IEEE Trans. Veh. Technol. 2011, 60, 1461-1469.

8. Liu, L.; Wang, L.Y.; Chen, Z.; Wang, C. Integrated system identification and state-of-charge estimation of battery systems. IEEE Trans. Energy Convers. 2013, 28, 12-23. [CrossRef]

9. Liu, X.; Chen, Z.; Zhang, C.; Wu, J. A novel temperature-compensated model for power Li-ion batteries with dual-particle-filter state of charge estimation. Appl. Energy 2014, 123, 263-272. [CrossRef]

10. Sun, F.; Xiong, R. A novel dual-scale cell state-of-charge estimation approach for series-connected battery pack used in electric vehicles. J. Power Sources 2015, 274, 582-594. [CrossRef]

11. Sun, F.; Xiong, R.; He, H. A systematic state-of-charge estimation framework for multi-cell battery pack in electric vehicles using bias correction technique. Appl. Energy 2016, 162, 1399-1409. [CrossRef]

12. Dong, G.; Wei, J.; Zhang, C.; Chen, Z. Online state of charge estimation and open circuit voltage hysteresis modeling of LiFePO 4 battery using invariant imbedding method. Appl. Energy 2016, 162, 163-171. [CrossRef]

13. Wang, Y.; Zhang, C.; Chen, Z. On-line battery state-of-charge estimation based on an integrated estimator. Appl. Energy 2015, 185, 2026-2032. [CrossRef]

14. Zhang, C.; Wang, L.Y.; Li, X. Robust and adaptive estimation of state of charge for lithium-ion batteries. IEEE Trans. Ind. Electron. 2015, 62, 4948-4957. [CrossRef]

15. Rahimi-Eichi, H.; Baronti, F.; Chow, M.Y. Online adaptive parameter identification and state-of-charge coestimation for lithium-polymer battery cells. IEEE Trans. Ind. Electron. 2014, 61, 2053-2061. [CrossRef]

16. Chen, Z.; Wang, L.Y.; Yin, G.; Wang, C. Accurate Probabilistic Characterization of Battery Estimates by Using Large Deviation Principles for Real-Time Battery Diagnosis. IEEE Trans. Energy Convers. 2013, 28, 860-870. [CrossRef] 
17. Zhang, H.; Pei, L.; Sun, J.; Song, K.; Lu, R.; Zhao, Y. Online Diagnosis for the Capacity Fade Fault of a Parallel-Connected Lithium Ion Battery Group. Energies 2016, 9, 387. [CrossRef]

18. Zheng, Y.; Han, X.; Lu, L.; Li, J.; Ouyang, M. Lithium ion battery pack power fade fault identification based on Shannon entropy in electric vehicles. J. Power Sources 2013, 223, 136-146. [CrossRef]

19. Satadru, D.; Ayalew, B. A diagnostic Scheme for Detection, Isolation and Estimation of Electrochemical Faults in Lithium-ion Cells. In Proceedings of the ASME Annual Dynamic Systems and Control Conference, Columbus, OH, USA, 28-30 October 2015; p. 43.

20. Dey, S.; Mohon, S.; Pisu, P.; Ayalew, B.; Onori, S. Online state and parameter estimation of Battery-Double Layer Capacitor Hybrid Energy Storage System. In Proceedings of the 54th IEEE Conference on Decision and Control, Osaka, Japan, 15-18 December 2015; pp. 676-681.

21. Jim, M.; Onori, S.; Rizzoni, G. Nonlinear Fault Detection and Isolation for a Lithium-Ion Battery Management System. In Proceedings of the ASME Dynamic Systems and Control Conference, Cambridge, MA, USA, 12-15 September 2010; pp. 607-614.

22. Zhou, D.H.; Su, Y.X.; Xi, Y.G.; Zhang, Z.J. Extension of Friedland's separate-bias estimation to randomly time-varying bias of nonlinear systems. IEEE Trans. Autom. Control 1993, 38, 1270-1273. [CrossRef]

23. Wang, X.X.; Zhao, L.; Xia, Q.X.; Hao, Y. Strong tracking filter based on unscented transformation. Control Decis. 2010, 25, 1063-1068.

24. Sun, G.Q.; Huang, M.Y.; Wei, Z.N.; Sun, Y.; Zang, H. Dynamic State Estimation for Synchronous Machines Based on Unscented Transformation of Strong Tracking Filter. Proc. CSEE 2016, 36, 615-623.

25. Hu, G.G.; Liu, Y.H.; Gao, S.S.; Yang, Y. Improved strong tracking UKF and its application in INS/GPS integrated navigation. J. Chin. Inert. Technol. 2014, 22, 634-639.

26. Zou, C.; Manzie, C.; Nešić, D. A Framework for Simplification of PDE-Based Lithium-Ion Battery Models. IEEE Trans. Control Syst. Technol. 2016, 24, 1594-1609. [CrossRef]

27. Chiang, C.J.; Yang, J.L.; Cheng, W.C. Temperature and state-of-charge estimation in ultracapacitors based on extended Kalman filter. J. Power Sources 2013, 234, 234-243. [CrossRef] 